\title{
TENDENCIAS DISCIPLINARES Y PROFESIONALES DE LA GEOGRAFÍA EN COSTA RICA: EL CASO DE LA UNIVERSIDAD NACIONAL
}

\author{
DISCIPLINARY AND PROFESSIONAL GEOGRAPHIC \\ TRENDS IN COSTA RICA: THE CASE OF THE \\ NATIONAL UNIVERSITY
}

\author{
Iliana Araya Ramírez ${ }^{1}$ \\ Universidad Nacional de Costa Rica
}

\begin{abstract}
RESUMEN
El objetivo del artículo consiste en examinar las tendencias disciplinarias y profesionales de la Geografía, así como discutir las principales relaciones entre el desarrollo disciplinar y profesional. La actualización del currículum de la carrera de Ciencias Geográficas con énfasis en Ordenamiento del Territorio, toma como referencia tales tendencias y las integra en una Geografía aplicada para responder a la necesidad de formar profesionales en geografía, que ofrezca soluciones en la ordenación del territorio tendiente a la equidad y el compromiso con el desarrollo nacional. Las áreas de desarrollo disciplinar y profesional de la carrera corresponden a Pensamiento, epistemología y enseñanza de la Geografía, Gestión y ordenamiento del territorio, Territorio y ambiente y Ciencia de la información geográfica.
\end{abstract}

Palabras clave: Tendencias disciplinares y profesionales en Geografía, epistemología de la geografía, territorio y ambiente, ciencias de la información geográfica, enseñanza de la geografía.

1 Académica e investigadora, Escuela de Ciencias Geográficas, Universidad Nacional de Costa Rica. Correo electrónico: ilianaraya@gmail.com

Fecha de recepción: 10 de setiembre de 2015

Fecha de aceptación: 24 de noviembre de 2015 
Iliana Araya Ramírez. Tendencias disciplinares y profesionales de la geografía en Costa Rica: el caso de la Universidad Nacional

DOI: http://dx.doi.org/10.15359/rgac.1-56.1

\begin{abstract}
The article aims at considering disciplinary and professional Geographic trends and discussing the main relationships between discipline and the professional development of Geography. Updating the curriculum of the Carrera de Ciencias Geográficas con énfasis en ordenamiento del territorio (Geographic Science Career with An Emphasis on Spatial Planning) is based on those trends and integrates them into an applied Geography to respond the needs of training professionals that provide spatial planning solutions aimed at the equity and commitment to national development. The disciplinary and professional development areas of the career correspond to Thinking, Epistemology and Geography Teaching, Land Use Planning and Management, Territory and Environment, and Geographic Information Science.
\end{abstract}

Keywords: Disciplinary and professional trends in Geography, epistemology of Geography, territory and environment, geographic information science, Geography teaching.

La dinámica transformación económico-social, cultural, ambiental y tecnológica en el contexto de globalización contemporánea impacta la disyuntiva ya existente entre la educación y las necesidades empresariales. La apertura económica derivada de los tratados de libre comercio exige un recurso humano con el desarrollo de capacidades, el dominio de técnicas, de habilidades y destrezas enfocados a la producción (Jiménez, 2008). Estos cambios globales demandan de la educación superior, reformas educativas que requieren modificación de las prácticas educativas y elaboración de nuevas propuestas de armonización curricular. De ahí que las interrogantes planteadas sobre la formación de los graduados y su vinculación con la actividad económica y productiva de los países son crecientes y la sociedad actual admite que la formación de profesionales se renueve en periodos inferiores a los cinco años con la capacidad de adaptación al contexto.

De esta manera, la formación de geógrafos implica el desafío de aportar algo significativo sobre las interacciones entre fenómenos del mundo natural y la sociedad; la complejidad en el análisis de la superficie terrestre y la diversidad e interrelación de fenómenos y procesos naturales y sociales. Asimismo, se precisa focalizar en la formación de expertos en temas regionales y en la ordenación del territorio que sean capaces de interpretar y explicar la compleja diversidad de áreas diferentes existentes en la tierra (Capel, 1998).

La actualización del currículum de la carrera de Ciencias Geográficas con énfasis en Ordenamiento del Territorio, toma como referencia las tendencias disciplinarias y profesionales de la Geografía. En el campo disciplinario se plantean nueve desafíos que debe enfrentar la Geografía 
en el ámbito latinoamericano, estos son: la globalización, la crisis ambiental, la desarticulación del tejido social, la alteración del orden político y territorial de producción del espacio, el desarrollo sostenible, la nueva cultura de planificación y gestión, la reconstrucción epistemológica del conocimiento, el avance y reconversión de las tecnologías de la información y la educación holística (Rodríguez. Citado por Carreto, Pérez, Reyes y Olmos, 2010).

En tanto las perspectivas profesionales que orientan los planes de estudio en la carrera de Ciencias Geográficas desde 1990 son coherentes con las reformas curriculares plasmadas en el Libro Blanco: título de grado en Geografía y Ordenación del Territorio del 2005, de la Agencia Nacional de Evaluación de la Calidad y Acreditación (ANECA). Los campos profesionales y las líneas de trabajo son el resultado de los estudios de seguimiento de graduados desde el 2004. El procedimiento metodológico consistió en la revisión de fuentes de información secundarias, aplicación de instrumentos y técnicas (cuestionarios, grupos focales, talleres, etc.), sistematización y análisis de resultados que se resumen en el presente artículo.

\section{La distinción conceptual entre la disciplina y la profesión}

La disciplina, por definición, constituye un campo de conocimientos que pertenecen a un ámbito cognoscitivo más amplio (las ciencias naturales, sociales, formales) que es desarrollada por un cuerpo académico y se insertan en el currículum como componente formativo (Hawes, 2010). En cambio, la profesión, en general, y de forma más específica, la actividad profesional del graduado en geografía, corresponde a la certificación que lo habilita para enfrentar problemas geográficos y proponer alternativas de ordenamiento y de planificación territorial.

Las disciplinas tienen objetos bien definidos y organizados de manera consistente respecto de su matriz teórica. En contraste, las profesiones se caracterizan por su interdisciplinariedad, dado que sus objetos no pueden ser entendidos fuera de la articulación de diversas disciplinas que el sujeto debe organizar para dar cuenta de la identidad profesional y enfrentar los problemas de su profesión (Hawes, 2009).

Las distinciones entre disciplina y profesión aparecen cuando se consideran la orientación de las mismas. En la primera es la generación de saberes desde la construcción teórica; en cambio, las profesiones "se orientan 
Iliana Araya Ramirez. Tendencias disciplinares y profesionales de la geografía en Costa Rica: el caso de la Universidad Nacional

DOI: http://dx.doi.org/10.15359/rgac.1-56.1

a la generación de soluciones en contextos y situaciones claramente identificables, contenidas en un tiempo y un espacio, en una cultura y una sociedad determinadas, en un entrecruzamiento de relaciones de la más variada naturaleza" (Hawes, 2009, p. 5). La segunda radica en que las disciplinas producen saberes descontextualizados en propuestas de carácter nomotética, tener forma de ley, apariencia de norma, de validez universal (Bunge. Citado por Hawes, 2009); no obstante, las profesiones tienen una orientación hacia lo idiosincrático, a lo específico de los problemas que atiende y a los saberes locales y contingentes. La tercera distinción se relaciona con el contexto de producción de ambos tipos de saber; el saber disciplinar ligado a la tradición de la ciencia, su proceso de producción puede asimilarse a un modelo de laboratorio; al contrario, el saber profesional se instala en un contexto de praxis, donde no se controlan de manera completa las variables y se genera un ambiente de incertidumbre (Hawes, 2009).

En síntesis, la distinción entre la disciplina y el campo profesional surgen con la inserción en el mundo del trabajo de los graduados. La disciplina geográfica genera un saber académico producto de la investigación, al igual que otras disciplinas "el contexto no es la realidad empírica sino que su referencia es siempre la construcción teórica" (Hawes, 2009, p. 5). En tanto, los profesionales buscan las soluciones a los problemas, los cuales acontecen en contextos reales, en un tiempo y espacio determinado y en sociedades y culturas específicas. Surge así la geografía aplicada, que busca integrar ambos saberes para ofrecer soluciones a los problemas ambientales de índole territorial. Esta orientación de la disciplina plantea desafíos para la formación de profesionales que aporten al desarrollo del país.

\section{La Geografía como disciplina}

El desarrollo de las ciencias en su conjunto colabora en la conformación de nuevas miradas para abordar los problemas geográficos e identificar problemáticas que en otra época desapercibidas o bien, no cumplían, pese a su importancia, con el estatuto de "problema científico". Este es el caso, por ejemplo, de la situación ambiental, la biotecnología, la bioética el avance de la tecnología que incluso ha alumbrado nuevas aristas de viejos problemas.

En ese contexto es necesario, también, apuntar la irrupción en el escenario de la investigación científica de nuevos actores sociales que demandan atención a sus problemáticas específicas, como por ejemplo, las 
minorías étnicas, las mujeres, los jóvenes, que hoy constituyen sujetos de estudio reconocidos como fuente de conocimiento válido. Todo esto obedece a importantes cambios paradigmáticos, que han puesto en entredicho las concepciones tradicionales de ciencia que se apoyaban en cosmovisiones cartesianas y newtonianas.

Estos cambios paradigmáticos de la evolución del pensamiento, en general, repercuten en la construcción teórica y metodológica de la geografía, y propician los espacios interdisciplinarios para la elaboración de instrumentos fundamentales en la definición y el estudio de nuevas problemáticas, lo que le permite a la Geografía redefinir su posición en el ámbito de las ciencias en la actualidad. A su vez, incide en la formación de profesionales en geografía que deben estar preparados para colaborar en la resolución de los problemas pertinentes a su objeto de estudio.

La geografía como disciplina estudia de forma integrada la interacción de los elementos esenciales de los sistemas biofísicos y socioeconómicos, con el propósito de entender la compleja diferenciación espacial resultante. Esta se caracteriza por grandes disparidades regionales que llevan a desiguales oportunidades en el plano socioeconómico, así como a serios trastornos y desequilibrios en el plano ecológico y medioambiental (Arce, Chan, Ocón y Segura, 2005).

El mapeo de la diferenciación regional y el conocimiento de las causas subyacentes es la piedra angular en que se apoya la disciplina geográfica para establecer los lineamientos de planificación y ordenamiento territorial. De esta manera, la geografía es una ciencia social y del territorio que, en la actualidad, recobra importancia para entender los procesos, los cambios y las problemáticas que se enfrentan en el mundo y en nuestro país. La planificación, sea esta sectorial o territorial, no cabe hacerse sin la consideración del factor geográfico (Vargas, 2010).

\section{Tendencias disciplinarias de la geografía en América Latina}

Las tendencias disciplinarias identificadas en América Latina responden a las temáticas abordadas en la investigación geográfica y que han sido expuestas en los Encuentros de Geógrafos de América Latina (EGAL). Los resultados de la investigación sobre las tendencias actuales de la geografía en América Latina identifican las orientaciones disciplinarias en el análisis del objeto de estudio. El análisis de frecuencias de los 
Iliana Araya Ramírez. Tendencias disciplinares y profesionales de la geografía en Costa Rica: el caso de la Universidad Nacional

DOI: http://dx.doi.org/10.15359/rgac.1-56.1

temas en las ponencias presentadas cada dos años, a partir del primer encuentro de Río Claro Brasil en 1987 al XV en la Habana, Cuba en el 2015, plantea cinco paradigmas temáticos tratados por los ponentes (Carreto. Citado por Carreto, Pérez, Reyes y Olmos, 2010; Carreto y González, 2013, Carreto, Rojas y González, 2015).

Figura 1: Paradigmas temáticos de la geografía en América Latina

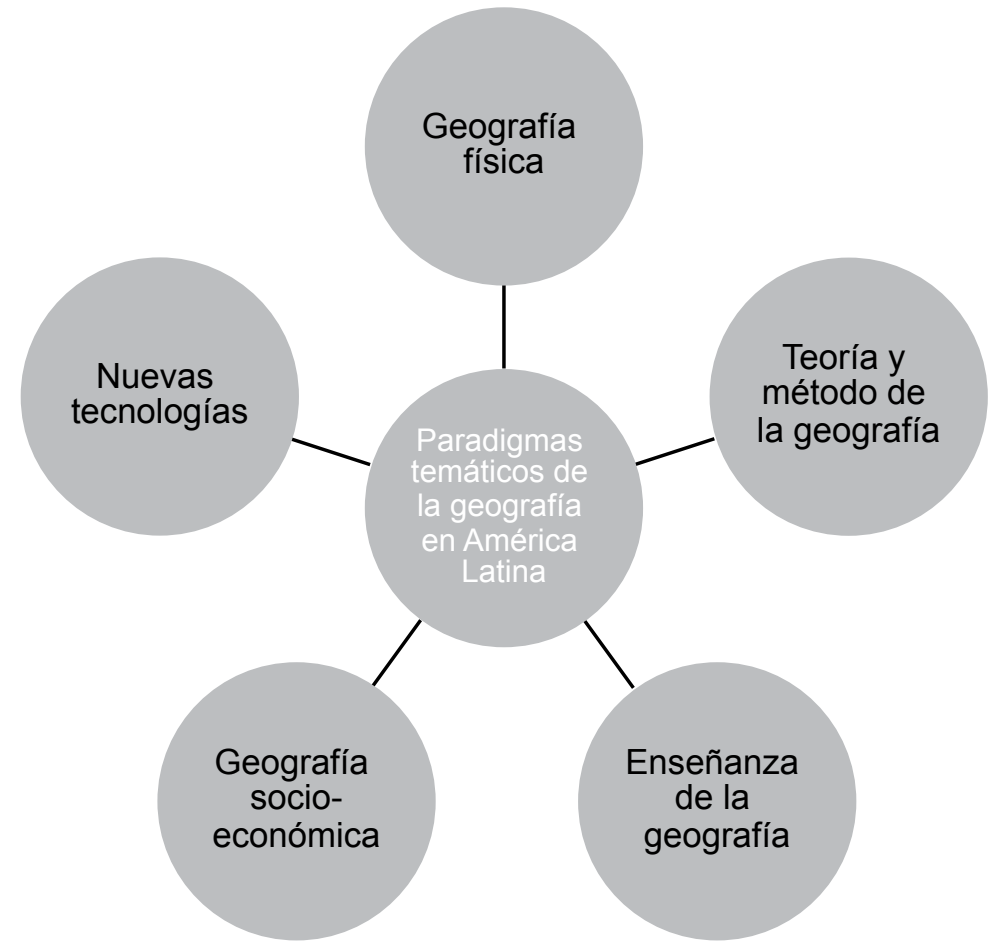

Fuente: Elaboración propia a partir de Carreto, Pérez, Reyes y Olmos, 2010; Carreto y González, 2013, Carreto, Rojas y González, 2015.

De los cinco paradigmas mencionados, la geografía socioeconómica domina como el paradigma significativo de la geografía latinoamericana, concentra una diversidad que se agrupa en orden de importancia en cuatro orientaciones disciplinarias: el análisis urbano-regional, la asimilación económica del espacio, el análisis socioespacial del territorio y el análisis político y cultural del espacio los investigados. 
La geografía física, la cual consolida la perspectiva social al tratamiento de los procesos ambientales en la ecología e impacto ambiental, los modelos y técnicas de la geografía física; así como el uso de los recursos ante la respuesta social a los riesgos naturales y de vulnerabilidad. Los paradigmas de Teoría y método de la geografía y la Enseñanza de la Geografía presentan con evidentes procesos de madurez teórico-metodológica y diferentes grados de desarrollo en el contexto latinoamericano; el primero incorpora los fundamentos teóricos, conceptuales y metodológicos de la geografía, el pensamiento geográfico e historia de la geografía y la investigación aplicada. En tanto la Enseñanza de la Geografía incluye la investigación educativa, la evaluación de programas de estudio en los niveles educativos: básico, medio y medio superior, nivel superior y de posgrado, la metodología para la enseñanza y el desempeño profesional. Finalmente, el paradigma tecnológico constituye un tópico emergente que se difunde en los diferentes campos de la disciplina geográfica, destacando el interés por generar su fundamentación teórico-metodológica a partir de la ciencia geográfica; desde los enfoques de la cartografía temática y automatizada, Sistemas de información geográfica (SIG) y la Teledetección (Carreto, Pérez, Reyes, Olmos, 2010; Carreto y González, 2013).

Las tendencias disciplinarias se engarzan con los desafíos para la geografía en el siglo XXI, que se presentaron en el Primer Encuentro Latinoamericano y Caribeño de Estudiantes de geografía por Mateo Rodríguez sobre "El mundo en el siglo XXI y los desafíos para la Geografía", en la Facultad de Geografía de la Universidad de la Habana, Cuba en el 2002. Rodríguez presentó nueve temas transversales que requieren atención en el ámbito geográfico y son considerados acordes a la circunstancia del territorio latinoamericano (Carreto, Pérez, Reyes y Olmos, 2010). Este autor plantea nueve desafíos que debe enfrentar la Geografía en relación con las tendencias mundiales; la globalización como primer desafío articula las transformaciones espaciales y territoriales a diferentes escalas en la construcción de un nuevo orden mundial; así como la especialización de los procesos globalizadores.

El segundo desafío es la crisis ambiental a nivel global enlazado con la geografía ambiental al tratar temas como el monitoreo de cambios de los sistemas ambientales, la medición de estados ambientales, el análisis de los factores desencadenadores de la crisis y la evaluación ambiental. 
Iliana Araya Ramírez. Tendencias disciplinares y profesionales de la geografía en Costa Rica: el caso de la Universidad Nacional

DOI: http://dx.doi.org/10.15359/rgac.1-56.1

Por su parte, el tercero corresponde a la desarticulación del tejido social abordado desde la Geografía social y cultural en la construcción de formas participativas en la gestión social, percepción acorde con los patrones culturales y territoriales; asimismo, la territorialidad e identidad. El cuarto, la alteración del orden político y territorial de producción del espacio concierne a la Geografía espacial mediante el monitoreo y evaluación de los cambios en las estructuras territoriales de producción espacial y la búsqueda de estructuras espaciales equilibradas y de estrategias espaciales por medio de la participación en las políticas públicas.

El desarrollo sostenible en coherencia con la Geografía del desarrollo es el quinto desafío, el cual alude a la adecuación de modelos y estilos de desarrollo a las diferentes escalas: global, regional, local, la medición de la sostenibilidad y la articulación espacial de estructuras económicas, sociales y ambientales para promover los procesos de desarrollo regional y local. El sexto, la nueva cultura de planificación y gestión corresponde a la Geografía aplicada para la planificación y la gestión e investiga la evaluación de escenarios en la evolución de los sistemas espaciales, la concepción prospectiva y métodos del pronóstico geográfico y la articulación entre sistemas ambientales, espaciales y territoriales así como la selección indicadores necesarios para la planificación.

La reconstrucción epistemológica del conocimiento en concordancia con la Filosofía y epistemología de la Geografía corresponde al séptimo desafío que se enmarca en los diálogos inter-intra y transdisciplinarios con otros saberes; la consolidación de categorías conceptuales como: espacio, territorio, paisaje, medio, región, la articulación entre la pluralidad de escuelas, un pensamiento humanista y cultural en la geografía y la confrontación entre la percepción científica y la popular de los sistemas geográficos. El octavo desafío recae en el avance y reconversión de las tecnologías de la Información en congruencia con la Tecnología de Información en Geografía (TIC) y el abordaje de la reconversión tecnológica, la cartografía computarizada, las ciencias de la Información geográfica, la producción cartográfica como forma de expresión y código de comunicación, el uso de sensores remotos.

Finalmente, la educación holística se articula con la educación geográfica en temáticas de educación ambiental, la integración de los componentes cognitivo, ético y aplicado, el encuentro inter y transdisciplinario, 
los modelos educativos, pedagógicos y didácticos de la geografía y la incorporación de las nuevas tecnologías informacionales en la enseñanza de la geografía.

Estos desafíos representan oportunidades para las propuestas de trabajo donde la disciplina asume un compromiso social con cada uno en su dimensión aplicada. El territorio se integra como un espacio geográfico organizado; ordenado reflejo de la acción meditada y concertada por el ser humano. Precisamente, el territorio enfrenta al profesional en Geografía a plantear alternativas para la resolución de los problemas concretos de ordenación, en los diferentes ámbitos y escalas.

\section{Tendencias disciplinarias de la Geografía en Costa Rica: el caso de la Universidad Nacional}

La Geografía de la Universidad Nacional sigue las tendencias internacionales, las cuales se agrupan en cuatro áreas estratégicas circunscritas al Plan Estratégico de la Facultad de la Ciencias de la Tierra y el Mar (2013-2017)

a) Pensamiento, epistemología y enseñanza de la Geografia (PEEG) Esta área investiga la evolución del objeto de estudio de la geografía de sus métodos, aplicaciones y su relación con otras ciencias y la cultura, integra los elementos de la sociedad, la naturaleza y las tecnologías en la relación espacio - tiempo. Las líneas de desarrollo temático para el quinquenio son:

a. epistemología y metodologías de la investigación científica en geografía b. investigación en epistemología de la enseñanza de la geografía c. Investigación multidisciplinaria y transdisciplinaria de los estudios geográficos.

Las interrogantes que guían esta área estratégica son ¿para qué se construye el conocimiento geográfico? De ahí que consideramos que la investigación constituye un proceso fundamental que fortalece y construye el conocimiento permitiendo la integración de la teoría y la práctica para contribuir a la solución de problemas. Otra interrogante se refiere a ¿cómo se construye el conocimiento geográfico? El interés radica en la aplicación, la contrastación y la evaluación de los métodos utilizados en 
Iliana Araya Ramirez. Tendencias disciplinares y profesionales de la geografía en Costa Rica: el caso de la Universidad Nacional

DOI: http://dx.doi.org/10.15359/rgac.1-56.1

las actividades científicas concretas; y establece un elemento operativo vinculado a la práctica investigativa. En nuestro caso, se refiere a la forma de obtener el conocimiento en geografía.

Por su parte, la enseñanza de la Geografía en el nivel medio de la educación, enfrenta el desafío de educar individuos con sólida formación y el desarrollo de competencias geográficas y ciudadanas con capacidad de observar, comprender e interpretar el mundo que les rodea y sus connotaciones sociales: globalización, cambio climático, desarrollo humano, diversidad (biológica, socio-económica y cultural) y el desarrollo sustentable.

\section{Gestión y ordenamiento del territorio (GOT)}

Esta área propone desarrollar un conocimiento transversal e interdisciplinario desde la perspectiva geográfica como ciencia aplicada. La gestión y el ordenamiento del territorio integra los componentes: culturales, ambientales, socioeconómicos, tecnológicos y político institucional. Asimismo, propone un conjunto de procedimientos e instrumentos técnicos, de carácter político-administrativo que incida en las políticas públicas para la toma de decisiones a nivel territorial y en diferentes ámbitos y escalas geográficas: regiones, provincias, cantones, distritos, zonas costeras, áreas protegidas, áreas urbanas, áreas rurales, cuencas hidrográficas y otras unidades especiales (Escuela de Ciencias Geográficas, 2014).

Las temáticas que abordan esta área están relacionadas con propuestas de modelos para diferentes ámbitos territoriales. Los procesos de gestión del territorio: formulación de planes territoriales, estrategias de desarrollo territorial, análisis y construcción de nuevos marcos reguladores y políticos. Los ámbitos y escalas territoriales de aplicación corresponden a zonas urbanas, rurales, costeras, montañosas, cuencas, y otras tipologías del territorio. El abordaje metodológico: conjugación de los procesos sociales, políticos y procesos técnicos con el enfoque ético de la Universidad Nacional. Así como la construcción y uso de la información pertinente para el ordenamiento e innovación en la valoración de los recursos del territorio (Escuela de Ciencias Geográficas, 2014).

\section{b) Territorio y ambiente ( $T A$ )}

Esta área estratégica articula los abordajes que realiza la geografía desde la perspectiva territorial de apropiación y uso que hacen las 
sociedades de los recursos y bienes. Enfocándose en la temática del riesgo por peligros naturales y antrópicos, el cambio climático, la gestión integral de cuencas hidrográficas, la gestión del agua, el estudio del paisaje, su manejo y conservación, la evaluación estratégica ambiental, la valoración económica, social y territorial y la gestión de residuos. Lo anterior para una planificación sustentable y eficiente del territorio que mejore la calidad de vida de los pobladores y potencie la conservación y restauración ecológica y cultural. Cada una de las temáticas mencionadas es abordada desde sus propias técnicas y marcos teórico-metodológicos (Escuela de Ciencias Geográficas, 2014).

\section{Los temas estratégicos}

- Gestión integral de cuencas hidrográficas y gestión del agua

- Gestión de riesgos por peligros naturales y antrópicos

- Conservación, paisaje y territorio

- Evaluación estratégica ambiental territorial y gestión de residuos

- Cambio climático y adaptación

- Valoración económica, social y territorial de bienes y recursos

\section{c) Ciencia de la información geográfica (CIG)}

La Ciencia de la Información Geográfica es un área cognitiva que integra un conjunto de disciplinas para el análisis de datos geográficos apoyados en herramientas tecnológicas, que conducen al modelado, análisis y representación espacial de la realidad. Esta área constituye un campo de la investigación, aplicado a procesos de ordenamiento territorial desembocando en propuestas curriculares para el fortalecimiento de la docencia, la investigación y la extensión.

Aborda temáticas como: a) Modelos y sistemas de información geográfica y teledetección b) Programación y aplicaciones geoespaciales, c) Base de datos y modelos relacionales d) Cartografía, e) Análisis de redes: análisis, conectividad y accesibilidad, f) Gerencia y gestión de proyectos en SIG, g) Análisis geográfico de mercados, h) Divulgación de productos cartográficos y comunicación virtual, i) Análisis espacial. 
Iliana Araya Ramírez. Tendencias disciplinares y profesionales de la geografía en Costa Rica: el caso de la Universidad Nacional

DOI: http://dx.doi.org/10.15359/rgac.1-56.1

\section{Tendencia de la Geografía profesional en América Latina}

Profesionales en Geografía de diferentes países latinoamericanos investigan el desarrollo de la profesión y muestran una preocupación compartida en el papel que debe jugar la universidad en la formación profesional. Diversos autores han publicado acerca de la geografía en América; En 2009, Muñiz y Boehm editan el libro Geography Education pan American Perspectives, el cual recoge la experiencia en algunos países latinoamericano y Estados Unidos; en este mismo año Sánchez y Liberali, compilaron La Geografia en América Latina: visión por países de la Unión geográfica de América Latina y el Centro Humboldt, estos autores recogen la experiencia latinoamericana en la geografía y sus enseñanza. En 2012, Pérez, Araya; Carreto, y Reyes, compilan La función social de la geografía en América Latina; estos autores procuran un abordaje acerca de ¿cuál es la función social de la disciplina? Esta perspectiva se enriquece tanto desde el campo profesional como desde la enseñanza.

Autores de Chile (Larrain y Hidalgo, 1993; Abálos, 2004), Argentina (Reboratti, 2001), Perú (Córdova, 2009), Ecuador (Hidalgo y Mayorga, 2009), Venezuela (Santiago, 2009), México (Sánchez y Ramos, 2009; Reyes, Pérez, y Carreto, B, 2012), Costa Rica (Quirós, 2009, Araya, 2010, 2011, 2012, Vargas, 2010); Panamá (Hernández, 2012), han documentado el desarrollo de la profesión. En el ámbito latinoamericano estos autores coinciden en los campos de trabajo del profesional en Geografía, los cuales se asocian a la investigación, la planificación territorial y sectorial, el diseño urbano, el transporte y la regeneración urbana, la planificación estratégica tanto pública como privada, la planificación del medio natural, los planes de expansión empresarial, el manejo de la cartografía automatizada, la aplicación de los sistemas de información geográfica y la participación en estudios de impacto ambiental. La planificación territorial y el manejo de recursos naturales son tareas tradicionales desarrolladas por estos profesionales. Al igual que la enseñanza de la geografía en los niveles medio y superior de la educación. En tanto, el manejo de la cartografía automatizada, la aplicación de los Sistemas de Información Geográfica en la planificación, los recursos naturales y el ordenamiento del territorio son tareas emergentes que se desarrollan con mayor fuerza después de la década del noventa (Araya, 2012). 
La incursión de los profesionales en Geografía en las áreas emergentes favorece la actualización de los planes de estudio acorde con las demandas sociales. Persiste una fuerte orientación a generar habilidades y capacidades en los campos de los sistemas de información geográfica, los métodos de representación cartográfica, la cartografía automatizada y una sólida formación en geografía física y humana. De esta forma, históricamente la geografía está al servicio de la sociedad con la finalidad de satisfacer las demandas emergentes.

Las áreas de desempeño del profesional en Geografía en los diferentes contextos corresponden a la situación costarricense. Este profesional tradicionalmente colabora en la propuesta de políticas públicas a nivel local y regional, en la ordenación de espacios naturales protegidos y la planificación urbana en el ámbito local. Asimismo, en grupos de investigación en las universidades o bien desde empresas de servicios especializadas (Vargas, 2010). De acuerdo con la opinión de los graduados entre 1973 y 2009 se interrogó acerca de ¿Cuál es el aporte del profesional en geografía a la sociedad costarricense? La planificación y el ordenamiento territorial se consideran el principal aporte, seguido de los recursos naturales y el ambiente y finalmente, gestión de las tecnologías de la información geográfica. El aporte del profesional en geografía formado en la Universidad Nacional consiste en la búsqueda de soluciones a problemas de índole territorial y ambiental, y su contribución al ordenamiento del territorio (Araya, 2012).

El alto nivel de compromiso social y ambiental es la piedra angular en la formación de los profesionales en Geografía, estos ofrecen una amplia perspectiva sobre las consecuencias sociales y ambientales producto de la relación indisociable entre la sociedad y el espacio geográfico. A diferencia de otros profesionales la formación holística que combina las ciencias naturales, exactas y sociales enriquece la perspectiva global e integradora de la Geografía; esto le permite al profesional en Geografía manejar su propio lenguaje, así como los métodos, las técnicas, las herramientas, los paradigmas y las escalas espacio-temporales de análisis que le son particulares (Araya, 2012) 
Iliana Araya Ramirez. Tendencias disciplinares y profesionales de la geografía en Costa Rica: el caso de la Universidad Nacional

DOI: http://dx.doi.org/10.15359/rgac.1-56.1

\section{Tendencia de la Geografía profesional en Costa Rica: el caso de la Universidad Nacional}

En el 2010, la carrera de Ciencias Geográficas con énfasis en ordenamiento territorial recibe la certificación de calidad otorgada por el Sistema de Nacional de Acreditación de la Educación Superior (SINAES) y da seguimiento a sus graduados de manera sistemática.

El Informe del proceso de autoevaluación (2010-2013) para ser sometido al Sistema Nacional de Acreditación de la Educación Superior para la carrera de Bachillerato y Licenciatura en Ciencias Geográficas con énfasis en Ordenamiento del Territorio examina las condiciones del mercado laboral del profesional en Ciencias Geográficas se exploran por medio de los estudios de seguimiento de graduados. El primer trabajo se denominó: "Inserción laboral y líneas de trabajo del geógrafo de la Universidad Nacional", realizado con la población graduada entre el 2002 y el 2008. Al analizar la tasa de general de ocupación de los graduados en labores afines con la Geografía en este período, los resultados mostraron que la tasa de colocación fue de un 89,00\%; del mismo modo, entre el 2005 y el 2008, se incrementó la cantidad de graduados que laboraban en el sector público de $50,00 \%$ a $70,00 \%$, aunque el porcentaje se contrajo en la categoría de trabajos por cuenta propia, pasando de 7,00\% a $0 \%$ (Quirós, Araya, Alfaro y Vargas, 2014).

Por otra parte, con la finalidad de responder a la interrogante: ¿Cuáles son las tareas que realizan los profesionales en geografía?, se definieron cuatro áreas de trabajo: ciencias de la información geográfica; paisaje, territorio y recursos naturales; gestión y ordenamiento del territorio, y enseñanza y divulgación de la geografía; éstas áreas se subdividieron en 16 líneas de trabajo, donde se encontró que el área de Ciencias de la Información Geográfica es la de mayor empleabilidad, dada su aplicación en otras áreas como ordenamiento y gestión territorial, y paisaje, territorio y recursos naturales

El segundo estudio: "Seguimiento de graduados como elemento de innovación curricular en la carrera de Ciencias Geográficas con énfasis en Ordenamiento del Territorio, Universidad Nacional, Costa Rica", abarca los graduados a nivel de bachillerato y licenciatura entre 1979 hasta el 2009. La tasa de colocación corresponde a $94,00 \%$, tasa que se incrementa a $100,00 \%$ con el grado de licenciatura; por su parte el $83,57 \%$ de esta 
población graduada se ubica en el sector público. Las líneas de trabajo de este conjunto de graduados siguen la misma orientación del primer estudio de seguimiento de graduados: Ciencias de la Información Geográfica, paisaje, territorio y recursos naturales, gestión y ordenamiento del territorio, y enseñanza y divulgación de la geografía. Los resultados de este trabajo coinciden con el "Seguimiento de la condición laboral de las personas graduadas 2000-2007 de las universidades costarricenses", que se componen del perfil de las personas graduadas, los aspectos académicos y la situación laboral (Quirós, Araya, Alfaro y Vargas, 2014).

El tercer estudio, "La función social de la geografía en Costa Rica", se centra en la caracterización del campo profesional y responde a la interrogante ¿Cuál es el aporte del profesional en geografía a la sociedad costarricense? La planificación y el ordenamiento territorial se consideran el principal aporte, seguido de los recursos naturales y el ambiente y finalmente, gestión de las tecnologías de la información geográfica. El aporte del profesional en geografía formado en la Universidad Nacional consiste en la búsqueda de soluciones a problemas de índole territorial y ambiental, y su contribución al ordenamiento del territorio.

En síntesis, los estudios de seguimiento de graduados, se han centrado en aspectos cuantitativos y cualitativos durante un periodo de 10 años. No obstante, la Carrera requiere profundizar en los espacios laborales de los graduados en las instituciones públicas y privadas donde la labor del profesional en geografía es poco conocida.

El actual estudio de seguimiento de graduados indica una contracción en el mercado laboral, consistente con el desempleo del país. Según el cuestionario aplicado a los graduados el 79,03 \% respondió que trabaja en tareas relacionadas con la carrera y un $20,93 \%$ que no lo hace. En este último caso, las razones por las cuales no trabajan, se relacionan con que, no encuentra trabajo en su campo, respondió el 44,44\%; continúa estudiando indicó el 33,33\%, y no encuentra trabajo en ningún campo el 22, $22 \%$, de estos últimos, el 66,67\% considera que no encuentra trabajo por la baja demanda de profesionales en su campo. El sector público continúa siendo el mayor empleador $61,76 \%$ (dentro de este, el 11,76\% corresponde a las municipalidades), seguido del sector privado, con $26,47 \%$; ONG el 2,94 $\%$ e igual cantidad, como consultor independiente). 
Iliana Araya Ramírez. Tendencias disciplinares y profesionales de la geografía en Costa Rica: el caso de la Universidad Nacional

DOI: http://dx.doi.org/10.15359/rgac.1-56.1

Como resultado de esta consulta en el año 2009 se procedió a identificar las tareas del profesional en Geografía de la Universidad Nacional en coherencia con las tendencias latinoamericanas y europeas, las cuales están documentadas en el artículo Inserción laboral y líneas de trabajo del geógrafo de la Universidad Nacional donde se identificaron y agruparon cuatro grandes áreas de trabajo y 16 líneas de trabajo. De esta forma, la identificación de la tendencias de la Geografía profesional en Costa Rica se propone a partir de la relación entre las áreas cognitivas, del plan estratégico de la Escuela de Ciencias Geográficas 2007-2012, con las líneas de trabajo de los graduados. Posteriormente, en 2013 se actualizan las áreas cognitivas en coherencia con Plan Estratégico de la Facultad de la Ciencias de la Tierra y el Mar (2013-2017).

El primer campo profesional donde se desempeñan los graduados y el mayor empleabilidad son las Ciencias de la Información Geográfica y las líneas de trabajo corresponden a la Cartografía, los Sistemas de Información Geográfica (manejo de datos), y la Teledetección, fotogrametría, sistemas de posicionamiento global. El segundo campo corresponde a Territorio y ambiente cuyas líneas de trabajo son Ordenamiento y gestión de espacios naturales, planes de emergencia y prevención de riesgos, estudios y auditorias, evaluación de impacto ambiental e inventarios de usos del suelo y recursos naturales.

El tercer campo profesional se centra en el énfasis de la carrera y se denomina Gestión y ordenamiento del territorio y las líneas son formulación de planes territoriales y gestión urbanística, política de suelo y trabajos catastrales, geomarketing: análisis de mercados y planes de expansión de actividades comerciales y estadísticas socioeconómicas y producción de información de base. Finalmente, el área de trabajo Pensamiento, epistemología y enseñanza de la Geografía se relaciona con la docencia e investigación y se está compuesta por Docencia y/o investigación universitaria, la docencia en enseñanza secundaria, la formación continua y enseñanza no formal y la educación ambiental (Araya, 2010).

Esta caracterización de la profesión establece la congruencia entre la formación dada en la educación superior y las áreas de trabajo en donde se inserta el profesional; las tareas tradicionales se vinculan a las temáticas ambientales, ordenación y planificación del territorio, las tareas emergentes están relacionadas con la aplicación de las tecnologías de la 
información geográfica y, las tareas decadentes se asocian con la enseñanza de la geografía en el III ciclo y la educación diversificada. Además, el carácter polivalente del profesional en geografía lo diferencia de otros profesionales y le otorga flexibilidad.

\section{Consideraciones finales}

La revisión de las tendencias disciplinares y profesionales de la geografía resultan fundamentales para orientar la propuesta curricular vigente, la cual no siempre responde a todos los requerimientos de la sociedad actual. La carrera de Ciencias Geográficas incorpora las tendencias internacionales en la disciplina, siempre en referencia a los problemas presentes en el contexto nacional y los principios orientadores de la Universidad Nacional. De esta forma, los temas relacionados con el ambiente y el territorio: el desarrollo sostenible, la degradación del paisaje, los riesgos naturales, los impactos ambientales, los modelos territoriales, la equidad espacial, la planificación territorial, entre otros, orientan la selección cultural de lo que debe ser enseñado y aprendido en la carrera.

No obstante, enfrenta grandes desafíos; en la sociedad de la información y comunicación las comunidades académicas y profesionales están encadenadas e interconectadas para generar información espacial necesaria en la toma de decisiones. Esta tarea requiere de profesionales con capacidad de comunicación, para el trabajo autónomo y una planificación adecuada del tiempo. En este sentido, el conocimiento técnico se convierte en un saber práctico ligado a la comunidad académica y profesional, ya que las líneas temáticas orientadoras del plan de estudio deben estar asociadas con la formación de profesionales que respondan a las demandas actuales.

Por su parte, el campo profesional de la geografía, también, es concordante con las tendencias del desarrollo profesional en América Latina y Europa; aun así mantiene rasgos particulares que la diferencian a nivel nacional e internacional, especialmente su carácter profesional en detrimento del académico. Las nuevas propuestas curriculares deben articular las tendencias disciplinares con la formación de profesionales que sean capaces de resolver los problemas de la sociedad. En la actualidad, Costa Rica enfrenta problemáticas claves: desigualdad social, crecimiento urbano y dificultades para el ordenamiento territorial, cambio climático, problemas demográficos característicos de una población que envejece. 
Iliana Araya Ramirez. Tendencias disciplinares y profesionales de la geografía en Costa Rica: el caso de la Universidad Nacional

DOI: http://dx.doi.org/10.15359/rgac.1-56.1

El profesional en geografía requiere una formación que lo faculte para la resolución de problemas en las áreas claves.

Las ciencias geográficas enfrentan grandes desafíos que deben ser coherentes con las nuevas propuestas curriculares; de un lado los avances tecnológicos requieren de una formación que dote al profesional de las herramientas necesarias para atender los problemas que enfrenta la profesión. De otro lado, la comprensión de la base disciplinaria posibilita la aplicación del conocimiento donde el graduado sea capaz de generar investigación desde su disciplina y articularse a equipos interdisciplinarios.

Finalmente, los estudios de seguimiento a graduados permiten monitorear la situación actual y gestionar acciones de forma que la oferta curricular pueda incidir en el contexto laboral. La divulgación del quehacer del profesional en geografía es una tarea urgente ¿Qué es un geógrafo? ¿Qué tareas realiza? ¿En cuáles ámbitos se puede desempeñar? Las respuestas a estas interrogantes forman parte de la divulgación de la profesión del geógrafo.

\section{Referencias}

Abálos, J. (2004). La geografía profesional en Chile: pasado y presente. Revista Polis de la Universidad Bolivariana, 3 (08). Recuperado de http://redalyc.uaemex.mx/redalyc/src/inicio/ArtPdfRed. jsp? iCve $=30500823$.

Agencia Nacional de Evaluación de la Calidad y Acreditación (ANECA). (2004). Libro Blanco Título de grado en Geografia y Ordenación del Territorio. Recuperado de http://www.uam.es/europea/lb_geografia.pdf.

Araya, F. (2010). Educación geográfica para la sustentabilidad. Editorial Universidad de Serena.

Araya, I. (2010). Inserción laboral y líneas de trabajo del geógrafo de la Universidad Nacional, Costa Rica. En Revista Geográfica de América Central. 44, 109-132.

Araya, I. (2011). Perfil por competencias para la carrera de Ciencias Geográficas con énfasis en Ordenamiento del Territorio, Universidad Nacional. Trabajo final de investigación aplicada sometido a la consideración de la Comisión del Programa de Estudios de Posgrado 
en Planificación Curricular para optar al grado y título de Maestría Profesional en Planificación Curricular Universidad de Costa Rica. Araya, I. (2012). La función social de la profesión geográfica en Costa Rica. En Pérez, B; Araya I; Carreto, F y Reyes, C (comp). La función social de la geografía en América Latina. Editorial Académica Española. ISBN 978-3-8473-6480-1.

Arce, R., Chan, M., Ocón, M., y Segura, O. (2005). Perfil del profesional en geografia. Manuscrito no publicado.

Carreto, F., Rojas, M., González, R. (2015). El observatorio geográfico de América Latina, repositorio de la producción científica de los EGAL en acceso abierto. En XV encuentro de Geógrafos de América Latina: por una América Latina unida y sustentable, La Habana, Cuba.

Carreto, F. y González, R. (2013). El Observatorio geográfico el espacio de difusión de los EGAL, 1987-2011. Ponencia presentada en 14 Encuentro de Geógrafos de América Latina (EGAL): reencuentro de saberes territoriales latinoamericanos, Lima, Perú.

Carreto, F., Pérez, B., Reyes, C. y Olmos, A. (2010). Propuesta para la creación del Departamento de Geografía Latinoamericana en la Facultad de Geografía de la UAEM. Ponencia presentada en $5^{\circ} \mathrm{COLO}$ QUIO GEOGRÁFICO SOBRE AMÉRICA LATINA. "Desafios que enfrenta América Latina en la globalización: una visión humanista y ambiental del espacio. Toluca, México.

Capel, H. (abril, 1998). Una geografía para el siglo XX. En Scripta Nova, Revista Electrónica de geografía y Ciencias Sociales, 2 (19). Recuperado de http://www.ub.es/geocrit/sn-19.htm

Córdova, H. (2009). "Geography Education in Perú." In Muñiz, O y Boehm R (editors) Geography Education pan American Perspectives. Ed Grosvenor Center for Geography Education. United State. Pp 269-295.

Facultad de Ciencias de la Tierra y el Mar. Plan estratégico 2013-2017. Universidad Nacional. Manuscrito no publicado.

Escuela de Ciencias Geográficas. (2014). Áreas cognitivas de Escuela de Ciencias Geográficas. Universidad Nacional, Costa Rica. Manuscrito no publicado.

Hawes, G. (2009). Las disciplinas, las profesiones y su enseñanza. Recuperado de http://www.gustavohawes.com/curriculum.htm. 
Iliana Araya Ramirez. Tendencias disciplinares y profesionales de la geografía en Costa Rica: el caso de la Universidad Nacional

DOI: http://dx.doi.org/10.15359/rgac.1-56.1

Hawes, G. (2010). El perfil de egreso. Recuperado: http://www.gustavohawes.com/curriculum.htm.

Hernández, G. (2012). Evaluación y actualidad de la Geografía en Panamá. Compendio desde la docencia, la investigación y el aporte institucional. En Pérez, B; Araya I; Carreto, F y Reyes, C (comp). La función social de la geografía en América Latina. Editorial Académica Española. ISBN 978-3-8473-6480-1.

Hidalgo, J. \& Mayorga, O. (2009). “Geography Education in Ecuador.” In Muñiz, O. y Boehm R. (editors) Geography Education pan American Perspectives. Ed. Grosvenor Center for Geography Education. United States. Pp 231-245.

Jiménez, J. (2008). Las competencias genéricas en el posgrado de la Universidad de Costa Rica. Trabajo final de investigación aplicada del posgrado de Evaluación Educativa. Universidad de Costa Rica.

Larrain, P. e Hidalgo. R. (1993). La geografía profesional en Chile en las puertas del Siglo XXI: realidades y desafíos. En Revista Geográfica de Chile Terra Australis, 37, 43-55. Recuperado: http://www.ub.es/ geocrit/sv-55.htm.

Muñiz, O. y Boehm, R. (editors). Geography Education pan American Perspectives. Ed. Grosvenor Center for Geography Education. United States. Pp 231-245.

Pérez, B., Araya, I., Carreto, F. y Reyes, C. (comp). (2012). La función social de la geografía en América Latina. Editorial Académica Española. ISBN 978-3-8473-6480-1.

Quirós, L. (2009). “Geography Education in Costa Rica”. In Muñiz, O. y Boehm R. (editors) Geography Education pan American Perspectives. Ed Grosvenor Center for Geography Education. United States. pp 205-230.

Quirós, L., Araya, A., Alfaro, D. y Vargas, A. (2014). Informe del proceso de autoevaluación (2010-2013) para ser sometido al Sistema Nacional de Acreditación de la Educación Superior para la carrera de Bachillerato y Licenciatura en Ciencias Geográficas con énfasis en Ordenamiento del Territorio. Universidad Nacional. Manuscrito no publicado.

Reyes, C., Pérez, B., y Carreto, B. (2012). La función social del geógrafo a partir de las tendencias de los trabajo de Tesis de la Facultad de Geografía de la UAEM. En Pérez, B., Araya, I., Carreto, F. y Reyes, 
C. (comp). (2012). La función social de la geografía en América Latina. Editorial Académica Española. ISBN 978-3-8473-6480-1.

Reboratti, C. (2001). La geografía profesional en Argentina. En Documents d'Anàlisi Geográfica, 39,119-130. Recuperado de http://ddd. uab.cat/record/1314/

Sánchez, A. \& Ramos, A. (2009). "Geography Education in México". In Muñiz, O y Boehm R (editors) Geography Education pan American Perspectives. Ed Grosvenor Center for Geography Education. United States. Pp 246-268.

Sánchez, A. y Liberali, A. (Comp). (2009). La Geografia en América Latina: visión por países. Unión geográfica de América Latina, UNAM, Centro Humboldt.

Santiago, J. (2009). "Geography Education in Venezuela". In Muñiz, O y Boehm R (editors) Geography Education pan American Perspectives. Ed Grosvenor Center for Geography Education. United States. Pp 321-342.

Vargas, G. (2010). La escuela de Geografía dentro de la Facultad de Ciencias Sociales de la Universidad de Costa Rica. En Revista Reflexiones, 89(1), 185-189. 